\title{
Il monastero di Bobbio in età altomedievale
}

Tesi di dottorato in "Archeologia e antichità post-classiche (secc. III-XI)", Università "La Sapienza" di Roma, XII ciclo, tutor G. Cantino Wataghin (Università del Piemonte Orientale "Amedeo Avogadro", sede di Vercelli), Marzo 2001

\section{Eleonora Destefanis}

\section{(2) OpenEdition Journals}

\section{Édition électronique}

URL : https://journals.openedition.org/cem/3562

DOI : $10.4000 /$ cem.3562

ISSN : 1954-3093

\section{Éditeur}

Centre d'études médiévales Saint-Germain d'Auxerre

Édition imprimée

Date de publication : 15 août 2003

ISSN : 1623-5770

\section{Référence électronique}

Eleonora Destefanis, « II monastero di Bobbio in età altomedievale », Bulletin du centre d'études médiévales d'Auxerre | BUCEMA [En ligne], 7 | 2003, mis en ligne le 29 novembre 2007, consulté le 22 septembre 2022. URL : http://journals.openedition.org/cem/3562 ; DOI : https://doi.org/10.4000/cem. 3562

Ce document a été généré automatiquement le 22 septembre 2022.

\section{c) (i) (2)}

Creative Commons - Attribution - Pas d'Utilisation Commerciale - Partage dans les Mêmes Conditions 4.0 International - CC BY-NC-SA 4.0

https://creativecommons.org/licenses/by-nc-sa/4.0/ 


\section{Il monastero di Bobbio in età altomedievale}

Tesi di dottorato in "Archeologia e antichità post-classiche (secc. III-XI)", Università "La Sapienza” di Roma, XII ciclo, tutor G. Cantino Wataghin (Università del Piemonte Orientale "Amedeo Avogadro", sede di Vercelli), Marzo 2001

\section{Eleonora Destefanis}

1 Il monastero di Bobbio, sorto sull'Appennino piacentino per iniziativa di S. Colombano e con il sostegno del re longobardo Agilulfo, ha conosciuto una lunga storia di studi che ne hanno messo di volta in volta in luce l'importanza come centro scrittorio e di raccolta di testi, il ruolo di primo piano quale interlocutore delle monarchie avvicendatesi nel corso dell'altomedioevo, la posizione di spicco nell'esperienza del monachesimo insulare sul Continente, riflessa da una cospicua produzione agiografica, che trova uno dei suoi momenti di maggior rilievo nella Vita di S. Colombano e dei suoi discepoli scritta dal monaco di Bobbio Giona.

2 Nell'ambito di una bibliografia molto consistente, di cui non si sono ricordati che alcuni filoni di ricerca, l'aspetto archeologico è sicuramente uno di quelli rimasti sinora più in ombra: l'attenzione per le strutture materiali del cenobio, la loro configurazione, funzione, evoluzione diacronica, ha spesso occupato un posto di minore rilevanza, anche in relazione ad una effettiva povertà di testimonianze, almeno a livello di edifici, dovuta principalmente ad una radicale ricostruzione dell'abbazia nel corso del XV secolo. Non diversa sorte è toccata ad una serie di materiali altomedievali (lastre funerarie, elementi di arredo liturgico, reliquiari, oggetti di pellegrinaggio), attualmente conservati nel Museo dell'Abbazia, ormai decontestualizzati, ma dotati di un forte potenziale informativo per la storia stessa del cenobio. Essi sono divenuti solo in minima parte oggetto di un'indagine scientifica, che metta in evidenza il rapporto con il complesso monastico, come parti integranti di esso.

3 Dalla considerazione della mancanza di uno studio organico attento al dato materiale nei suoi vari aspetti, e nell'intento di ricostruire nel modo più puntuale possibile il contesto globale in cui il cenobio si sviluppa nel corso dell'altomedioevo, ha preso le 
mosse la tesi di dottorato che si presenta sinteticamente in questa sede ${ }^{1}$, ora in buona parte pubblicata in: Eleonora DESTEFANIS, Il monastero di Bobbio in età altomedievale, Firenze, All'Insegna del Giglio, 2002 [Ricerche di Archeologia altomedievale e medievale, 27]. In stretta interazione con l'analisi di tutte le testimonianze materiali pertinenti alla vita dell'abbazia e riconducibili al periodo esaminato, l'indagine sul territorio montano in cui l'ente religioso si inserisce ha rappresentato un filo conduttore del lavoro, nella convinzione di poter apprezzare appieno la storia del monastero soltanto ricostruendo i suoi rapporti con gli ambiti più o meno vasti con cui esso ebbe a confrontarsi, con i territori su cui l'impatto della presenza bobbiese si avvertì in misura spesso incisiva.

4 Il cenobio sorge in una posizione particolarmente favorevole dal punto di vista viario, in una zona di intersezione di percorsi di notevole rilievo nel sistema itinerario dell'Italia centro-settentrionale, lungo la strada che collegava Piacenza (e attraverso questa città la pianura padana e da ultimo i principali valichi alpini) con Genova (e quindi con il mare da un lato, ma anche con l'Italia centrale e con Roma). Nel contempo, attraverso le valli contermini ad Occidente, si snodavano i percorsi verso Tortona e soprattutto Pavia, che andavano a confluire nella zona di Bobbio. Accanto agli assi di viabilità principale si è rintracciata una fitta rete di tracciati di portata locale, secondari rispetto alle grandi vie di comunicazione, ma fondamentali per assicurare i collegamenti intervallivi, vero tessuto connettivo tra gli insediamenti che già in età romana - senza considerare il periodo pre e protostorico - si distribuiscono sui versanti appenninici del vasto comprensorio bobbiese.

5 Dalla carta archeologica della zona, redatta sulla base dell'edito e soprattutto delle informazioni sui rinvenimenti per lo più di superficie ricavabili dagli Archivi della Soprintendenza per i Beni Archeologici dell'Emilia-Romagna e dai Gruppi archeologici operanti sul territorio, emerge con chiarezza l'articolazione insediativa che sin dal periodo romano caratterizza questo ambito montano: ad un'occupazione sparsa, organizzata in siti di maggior tenore qualitativo e in nuclei di livello più modesto, forse semplici fattorie o centri produttivi, si affiancavano probabilmente abitati di tipo accentrato, la cui percezione archeologica tuttavia, risulta al momento suggerita soltanto da alcuni indizi. A quest'ultima tipologia insediativa non è da escludere che si possa ricondurre il caso stesso di Bobbio, per cui la posizione itineraria all'incrocio di importanti percorsi, il controllo su un punto di attraversamento del fiume Trebbia, un'epigrafe forse riferibile ad una magistratura vicana, la presenza di acque salse già sfruttate in antico, potrebbero essere indici di un accentramento dell'insediamento.

Il centro bobbiese compare per la prima volta in modo esplicito nell'opera agiografica di Giona, il quale dà notizia dell'esistenza sul luogo ove sorgerà il monastero di una basilica Sancti Petri ormai in abbandono (semiruta), che Colombano avrebbe trovato al suo arrivo sul posto. La presenza dell'edificio di culto si inserisce nel più vasto problema della cristianizzazione delle campagne, di cui tuttavia, per quanto concerne soprattutto l'area montana piacentina, restano oscuri modalità, tempi, strumenti di diffusione nonché soggetti promotori, poiché, accanto all'azione più ovvia del vescovo piacentino, non si esclude possa avere avuto qualche parte anche l'ordinario di Tortona.

7 In assenza al momento di tracce strutturali ascrivibili al periodo altomedievale, in quanto i lacerti di muratura più antichi, sopravvissuti alla ricostruzione rinascimentale, sembrano riconducibili al tardo X-inizi XI secolo, sono soprattutto le 
fonti scritte a risultare di particolare interesse nella prospettiva di una ricostruzione della configurazione del centro monastico, sin dalle prime fasi. L'opera di Giona lascia intravedere, entro un recinto che si va strutturando sin dall'epoca dell'abate Attala, primo successore di Colombano (che septa monastirii densat), l'edificio di culto che si provvede a rinnovare e le cellulae ove risiedono i religiosi. La vita quotidiana all'interno del centro monastico integra ai momenti di preghiera lo studio e le attività pratiche: in più fonti (ivi comprese notazioni su alcuni manoscritti bobbiesi) è ricordata la cura $\mathrm{e}$ l'attenzione per i libri conservati in apposite arcae, sorte di armadi, forse primo nucleo di una biblioteca, poi sicuramente attestata in periodo carolingio. La presenza di laboratori artigianali è suggerita dalla stessa opera di Giona, in cui compaiono allusioni a stalle, a locali per il confezionamento delle calzature, a depositi per il ricovero degli attrezzi agricoli o per lo stoccaggio dei cereali coltivati nelle terre circostanti e trasformati nel mulino monastico.

Il quadro si articola ulteriormente con il IX secolo, quando le fonti descrivono un cenobio complesso ed articolato, con una pluralità di edifici dalle molteplici funzioni, dalla chiesa, ai laboratori artigianali, al refettorio, al dormitorio, ai locali di assistenza e di ospitalità, oltre che alla biblioteca e allo scriptorium, in linea con gli assetti noti per le grandi abbazie carolinge.

In attesa di scavi futuri, che con ogni probabilità sostanzieranno il panorama configurato sulla base delle fonti scritte, alcune testimonianze materiali contribuiscono a riflettere anche l'alto livello qualitativo che doveva contraddistinguere il cenobio: le lastre funerarie dell'VIII secolo ad esempio, prima fra tutte quella dell'episcopus irlandese Cumiano, rappresentano non soltanto una delle espressioni più significative di tutta la produzione scultorea longobarda, ma restituiscono anche la dimensione e l'importanza degli stretti rapporti intercorrenti tra il cenobio e la corte pavese, nel cui ambito culturale e produttivo tali manufatti trovano la loro logica collocazione. Parimenti, le componenti di arredo liturgico di età carolingia, ascrivibili a elementi di recinzione presbiteriale (plutei, pilastrini, colonnine, forse di pergula), o a antependia di altare o forse in un caso a recinti a protezione di una sepoltura, così come gli elementi di decorazione architettonica (capitelli, forse per aperture polilobate), costituiscono, seppur in misura molto frammentaria, dei tasselli per la ricostruzione dell'edificio di culto.

Su tali manufatti, e soprattutto sulle non poche lastre conservate per intero o in misura significativa, è stato condotto uno studio volto a sviscerare il potenziale informativo in essi contenuto, anche al di là delle considerazioni di tipo stilistico, iconografico e funzionale. In particolare, attraverso l'uso della grafica computerizzata, è stato spesso possibile risalire agli schemi compositivi di base ed in qualche caso avanzare qualche proposta, almeno a livello di ipotesi di lavoro, sul modo di procedere adottato dai lapicidi che le realizzarono: sembra cioè affermarsi un procedere per sezioni progressive, spesso modulari, in cui, sulla trama di fondo costituita da assi portanti fondamentali, si innestano variazioni apportate dall'esecutore, che spesso, riproducendo un modulo di base, dà prova di muoversi con disinvoltura, in una compenetrazione continua e dinamica tra uno schema geometrico rigoroso - ma non rigido - e una certa libertà di interpretazione.

11 Il monastero, la cui configurazione anche materiale va progressivamente mutando nel corso dell'altomedioevo, diventa sempre più il vero punto di riferimento per il territorio circostante, anche grazie ad una rete di proprietà che si distribuiscono in 
modo talora molto capillare su un raggio piuttosto vasto. La documentazione scritta consente di seguire la costituzione del patrimonio bobbiese, che, dalla valle del Trebbia dove il monastero sorge, si espande nelle valli appenniniche fino a raggiungere la pianura padana, sino alla regione gardesana da un lato e la Liguria costiera (già all'inizio dell'età carolingia) e la Toscana settentrionale dall'altro.

Proprio attraverso l'insieme di beni e diritti su aree anche molto lontane dal centro monastico, questo, con un'efficiente gestione dei possedimenti, che trova nel sistema curtense una delle sue più efficaci espressioni, controlla lo sfruttamento di risorse differenziate e ottimizza l'articolazione produttiva, organizzando la convergenza al monastero di prodotti, poi ridistribuiti nel modo più opportuno.

Bobbio riesce nel contempo ad attuare strategie differenti nell'occupazione e nel controllo dei territori ad esso legati: lo studio di due aree-campione in cui il cenobio dispone di vari possedimenti - le valli verso Ovest e Nord-Ovest (Tidone, Tidoncello, Staffora) e quelle verso Sud-Est (Ceno e Taro) - ha dimostrato come da un lato i possedimenti monastici si inseriscano frequentemente in zone già sfruttate in età romana, sia dal punto di vista economico che da quello itinerario, dall'altro riflettano precise scelte e strategie di espansione, diversificate a seconda delle situazioni specifiche e del tipo di confronto/scontro con altri enti ecclesiastici o potentati laici con interessi sulle stesse aree. Nel caso del territorio a Sud-Est, ad esempio, il cenobio, impossibilitato dalla presenza di altri soggetti di potere ad estendere il proprio controllo fondiario in modo omogeneo, genera un patrimonio non contiguo, focalizzato principalmente su alcuni centri di notevole importanza itineraria, mentre assume un comportamento di ben maggiore radicamento a livello di distribuzione delle proprietà in zone in cui esso è meno ostacolato.

14 Anche per quanto concerne la vita religiosa, il monastero diviene ben presto un punto di riferimento per il territorio, sia direttamente, sia indirettamente, attraverso le numerose dipendenze, alcune delle quali sono ricordate almeno dal IX secolo come plebes, con una funzione dunque di inquadramento ecclesiastico delle popolazioni delle campagne. In altri casi, e soprattutto in corrispondenza di centri ubicati lungo vie di comunicazione, il cenobio dispone di xenodochia per il sostentamento dei poveri e dei viandanti, in particolare di pellegrini, non solo irlandesi, alla cui accoglienza è del resto specificamente preposto un ospizio nella città di Piacenza.

Il monastero stesso diviene, sin dall'indomani della fondazione, un centro di pellegrinaggio, grazie alla venerazione che ben presto si coagula intorno alla tomba di S. Colombano estendendosi poi nel corso del tempo al culto dei protoabati. Accanto alle fonti scritte, in cui sono abbastanza numerose le menzioni di pellegrinaggi a Bobbio, anche da regioni piuttosto lontane, prime fra tutte il mondo insulare, le attestazioni materiali ancora oggi conservate nel Museo dell'Abbazia, rendono testimonianza del ruolo svolto dal monastero sin dal VII secolo come tappa o talora anche come meta di viaggi devozionali, spesso in direzione di Roma o di ritorno da essa oppure dai grandi santuari del Mediterraneo, soprattutto orientale. Oltre ad oggetti molto noti, quali le celebri ampolle in lega di piombo e stagno, che si ritengono dono della regina Teodolinda al monastero, ma che con una certa probabilità potrebbero essere inquadrate nel flusso devozionale interessante l'abbazia, il sito conserva numerose euloge e medaglioni di terracotta, provenienti dai luoghi santi della Palestina e della Siria, talora provvisti di cestini in fibre vegetali per il trasporto, nonché reliquiari lignei di provenienza verosimilmente transalpina, un reliquiario metallico di produzione 
insulare, stoffe, ampolline vitree di cui è stato possibile rintracciare i tappi lignei di chiusura, ed altri piccoli oggetti, segno di una devozione che trova nel monastero un punto forte e che allarga enormemente gli orizzonti del "territorio" bobbiese.

\section{NOTES}

1.Essa è organizzata in due volumi, l'uno di testo, l'altro (Schede) costituito da una duplice schedatura relativa ai rinvenimenti nella valle del Trebbia e delle valli contermini tra età romana e altomedioevo e ai materiali scultorei altomedievali per lo più conservati nel Museo dell'Abbazia; ad essi si aggiunge un terzo volume (I materiali scultorei dell'Abbazia. Analisi compositiva. Documentazione), comprendente le tavole relative ai singoli elementi scultorei, illustranti gli schemi compositivi rintracciati come griglie di riferimento per il decoro.

\section{INDEX}

Mots-clés : monastère

Index géographique : Italie/Bobbio 